\title{
TENSOR PRODUCT WEIGHT MODULES OVER THE VIRASORO ALGEBRA
}

\author{
HONGJIA CHEN, XIANGQIAN GUO AND KAIMING ZHAO
}

\begin{abstract}
The tensor product of highest weight modules with intermediate series modules over the Virasoro algebra was discussed by Zhang $\mathrm{Z}$ in 1997. Since then the irreducibility problem for the tensor products has been open. In this paper, we determine the necessary and sufficient conditions for these tensor products to be simple. From non-simple tensor products, we can get other interesting simple Virasoro modules. We also obtain that any two such tensor products are isomorphic if and only if the corresponding highest weight modules and intermediate series modules are isomorphic respectively. Our method is to develop a "shifting technique" and to widely use Feigin-Fuchs' Theorem on singular vectors of Verma modules over the Virasoro algebra.
\end{abstract}

Keywords: Virasoro algebra, highest weight module, intermediate series module, simple module.

AMS classification: 17B10, 17B20, 17B65, 17B66, 17B68.

\section{INTRODUCTION}

We denote by $\mathbb{Z}, \mathbb{Z}_{+}, \mathbb{N}$ and $\mathbb{C}$ the sets of all integers, nonnegative integers, positive integers and complex numbers, respectively.

The Virasoro algebra Vir is an infinite dimensional Lie algebra over the complex numbers $\mathbb{C}$, with basis $\left\{d_{n}, C \mid n \in \mathbb{Z}\right\}$ and defining relations

$$
\begin{gathered}
{\left[d_{m}, d_{n}\right]=(n-m) d_{n+m}+\delta_{n,-m} \frac{m^{3}-m}{12} C, \quad m, n \in \mathbb{Z},} \\
{\left[C, d_{m}\right]=0, \quad m \in \mathbb{Z},}
\end{gathered}
$$

which is the universal central extension of the so-called infinite dimensional Witt algebra. The algebra Vir is one of the most important Lie algebras both in mathematics and in mathematical physics, see for example [IK, [KR] and references therein. In particular, it has been widely used in quantum physics [GO], conformal field theory [DMS], Kac-Moody algebras [K, $\mathrm{MoP}$, vertex operator algebras [DMZ, FZ], and so on.

The representation theory on the Virasoro algebra has been attracting a lot of attentions from mathematicians and physicists. There are 
two classical families of simple Harish-Chandra Vir-modules: highest (lowest) weight modules (completely described in $[\mathrm{FF}$ ) and the socalled intermediate series modules. In [M] it is shown that these two families exhaust all simple Harish-Chandra modules. In MZ1 it is even shown that the above modules exhaust all simple weight modules admitting a nonzero finite dimensional weight space. Now mathematicians have shifted their attentions to non-Harish-Chandra simple modules, mainly simple weight modules with infinitely dimensional weight spaces and non-weight modules.

As for simple weight modules over Vir with infinitely dimensional weight spaces, the earliest examples were constructed from tensor products of simple highest weight modules and intermediate series modules ([Z]) in 1997. Later a 4-parameter family of simple weight modules with infinitely dimensional weight spaces were given in [CM] in 2001 . Recently in [LLZ another huge class of simple modules with infinitely dimensional weight spaces were obtained using non-weight modules introduced in [MZ2].

At the same time for the last decade, various families of nonweight simple Virasoro modules were studied in [FJK, GLZ, LGZ, LZ, Y, MW, OW]. These (except the modules in [LZ and [MW]) are basically various versions of Whittaker modules constructed using different tricks. In particular, all the above Whittaker modules and even more were described in a uniform way in MZ2.

In the paper [Z], Zhang considered the tensor products of simple highest weight modules with simple intermediate series modules, and he provided some sufficient conditions for the tensor products to be simple. Since then the irreducibility problem for the tensor products has been open. It was even not known in [Z] whether there was a non-simple tensor product when the highest weight module is not a Verma module. Our purpose in the present paper is to completely solve this problem. More precisely we determine the necessary and sufficient conditions for such tensor products to be simple, using two polynomials obtained from the singular vectors of the original highest weight modules. Moreover we can determine the conditions for two of these tensor product modules to be isomorphic. We remark that the tensor products of intermediate series modules over the Virasoro algebra never gives irreducible modules [Zk].

To describe our results, we recall some notations for the Virasoro algebra and its modules. We first define the modules of intermediate series $V_{\alpha, \beta}$ for any $\alpha, \beta \in \mathbb{C}$. As a vector space $V_{\alpha, \beta}=\oplus_{n \in \mathbb{Z}} \mathbb{C} v_{n}$ and the action of Vir on $V_{\alpha, \beta}$ is given by

$$
d_{m} \cdot v_{n}=(\alpha+n+m \beta) v_{m+n}, \quad C \cdot v_{n}=0, \forall m, n \in \mathbb{Z} .
$$


It is well known that $V_{\alpha, \beta} \cong V_{\alpha+n, \beta}$, and $V_{\alpha, 0} \cong V_{\alpha, 1}$ if $\alpha \notin \mathbb{Z}$. It is also well known that $V_{\alpha, \beta}$ is simple if and only if $\alpha \notin \mathbb{Z}$ or $\beta \notin\{0,1\}$. Moreover $V_{0,0}$ has a unique nonzero proper submodule $\mathbb{C} v_{0}$ and we denote $V_{0,0}^{\prime}=V_{0,0} / \mathbb{C} v_{0} ; V_{0,1}$ has a unique nonzero proper submodule $V_{0,1}^{\prime}=\sum_{i \neq 0} \mathbb{C} v_{i}$ and $V_{0,0}^{\prime} \cong V_{0,1}^{\prime}$. For convenience we will denote $V_{\alpha, \beta}^{\prime}=$ $V_{\alpha, \beta}$ whenever $V_{\alpha, \beta}$ is simple. We remark that $V_{\alpha, 0}^{\prime} \cong V_{\alpha, 1}^{\prime}$ for all $\alpha \in \mathbb{C}$.

To avoid repetition, throughout this paper when we write $V_{\alpha, \beta}^{\prime}$ we always assume that $0 \leq \mathfrak{R e} \alpha<1$ and $\beta \neq 1$, where $\mathfrak{R e} \alpha$ is the real part of $\alpha$.

We recall that a Virasoro module $V$ is a weight module if it is the sum of all its weight spaces $V_{\lambda}=\left\{v \in V \mid d_{0} v=\lambda v\right\}$ for some $\lambda \in \mathbb{C}$. Vectors in $V_{\lambda}$ are call weight vectors. An element $x$ in the universal enveloping algebra $U(\mathrm{Vir})$ is called homogeneous if $\left[d_{0}, x\right]=m x$ for some $m \in \mathbb{Z}$ and $m$ is called the degree of $x$, denoted by $\operatorname{deg}(x)=m$. Let $U$ (Vir $)_{m}$ be the subspace consisting of all elements of degree $m$.

Next we define the highest weight modules. Denote $\operatorname{Vir}_{ \pm}=\sum_{i \in \mathbb{N}} \mathbb{C} d_{ \pm i}$. For any $h, c \in \mathbb{C}$, we let $\mathbb{C} u$ be the 1-dimensional module over the subalgebra $\operatorname{Vir}_{+} \oplus \mathbb{C} d_{0} \oplus \mathbb{C} C$ defined by

$$
\mathrm{Vir}_{+} u=0, d_{0} u=h u, \text { and } C u=c u \text {. }
$$

Then we get the induced Vir-module, called Verma module:

$$
M(c, h)=U(\operatorname{Vir}) \bigotimes_{U\left(\operatorname{Vir}_{+} \oplus \mathbb{C} d_{0} \oplus \mathbb{C} C\right)} \mathbb{C} u .
$$

Any nonzero quotient module of $M(c, h)$ is called a highest weight module with highest weight $(c, h)$.

It is well known that the Verma module $M(c, h)$ has a unique maximal submodule $J(c, h)$ and the corresponding simple quotient module is denoted by $V(c, h)$. A nonzero weight vector $u^{\prime} \in M(c, h)$ is called a singular vector if $\operatorname{Vir}_{+} u^{\prime}=0$. It is clear that $J(c, h)$ is generated by all singular vectors in $M(c, h)$ not contained in $\mathbb{C} u$, and that $M(c, h)=V(c, h)$ if and only if $M(c, h)$ does not contain any other singular vectors besides $\mathbb{C} u$. In $[\mathrm{FF}$ (or in [A] which is a refined form) the singular vectors are described explicitly. In particular, $J(c, h)$ can be generated by at most two singular vectors. If $J(c, h)$ is generated by two singular vectors, we can find homogeneous $Q_{1}, Q_{2} \in U\left(\mathrm{Vir}_{-}\right)$such that $J(c, h)=U\left(\mathrm{Vir}_{-}\right) Q_{1} u+U\left(\mathrm{Vir}_{-}\right) Q_{2} u$. If $J(c, h)$ is generated by one singular vector, we can find the unique $Q_{1} \in U\left(\mathrm{Vir}_{-}\right)$up to a scalar multiple such that $J(c, h)=U\left(\operatorname{Vir}_{-}\right) Q_{1} u$; in which case we set $Q_{2}=Q_{1}$ for convenience. When $M(c, h)=V(c, h)$ we set $Q_{2}=Q_{1}=0$. 
Fix any $c, h, \alpha, \beta \in \mathbb{C}$ with $0 \leq \mathfrak{R e} \alpha<1$ and $\beta \neq 1$. For any $n \in \mathbb{Z}$, we have a linear map $\varphi_{n}$ from $U\left(\right.$ Vir $\left._{-}\right)$to $\mathbb{C}$ defined by

$$
\varphi_{n}\left(d_{-k_{r}} \cdots d_{-k_{1}}\right)=\prod_{j=1}^{r}\left(k_{j} \beta-\alpha-n-\sum_{i=1}^{j} k_{i}\right),
$$

for all $d_{-k_{r}} \cdots d_{-k_{1}} \in U\left(\operatorname{Vir}_{-}\right)$. For more details of this map see Sect.2.

For any integer $n$ let $W^{(n)}$ be the submodule of $V(c, h) \otimes V_{\alpha, \beta}^{\prime}$ generated by $u \otimes v_{i}, i>n$. Then our main results can be stated as

Theorem 1. Let $c, h, \alpha, \beta \in \mathbb{C}$ with $0 \leq \mathfrak{R e} \alpha<1$ and $\beta \neq 1$.

(a) $V(c, h) \otimes V_{\alpha, \beta}^{\prime}$ is simple if and only if there is no (nonzero if $(\alpha, \beta)=$ $(0,0))$ integer $n$ such that $\varphi_{n}\left(Q_{1}\right)=\varphi_{n}\left(Q_{2}\right)=0$.

(b) If $n$ is the maximal integer (nonzero if $(\alpha, \beta)=(0,0)$ ) with $\varphi_{n}\left(Q_{1}\right)=$ $\varphi_{n}\left(Q_{2}\right)=0$, then $W^{(n)}$ is the unique simple submodule and all its nonzero weight spaces are infinite-dimensional.

Theorem 2. Let $V$ and $V^{\prime}$ be any two highest weight modules (not necessarily simple) of the Virasoro algebra, and let $\alpha, \beta, \alpha_{1}, \beta_{1} \in \mathbb{C}$. Then $V \otimes V_{\alpha, \beta}^{\prime} \cong V^{\prime} \otimes V_{\alpha_{1}, \beta_{1}}^{\prime}$ if and only if $V \cong V^{\prime}$ and $V_{\alpha, \beta}^{\prime} \cong V_{\alpha_{1}, \beta_{1}}^{\prime}$.

As a direct consequence we have

Theorem 3. Let $c, h, \alpha, \beta, c_{1}, h_{1}, \alpha_{1}, \beta_{1} \in \mathbb{C}$ with $0 \leq \mathfrak{R e} \alpha, \mathfrak{R e} \alpha_{1}<1$ and $\beta, \beta_{1} \neq 1$. Then $V(c, h) \otimes V_{\alpha, \beta}^{\prime} \cong V\left(c_{1}, h_{1}\right) \otimes V_{\alpha_{1}, \beta_{1}}^{\prime}$ if and only if $c_{1}=c, h_{1}=h, \alpha_{1}=\alpha$ and $\beta_{1}=\beta$.

The paper is organized as follows. In Sect.2, we prove Theorem 1. The main method is to develop a "shifting technique" and to widely use Feigin-Fuchs' Theorem on singular vectors of Verma modules over the Virasoro algebra. Theorem 2 is proved in Sect.3, and we show that the simple tensor products are not isomorphic to other known simple weight modules. In the last section we give several examples to illustrate our results. In particular, when $V(c, h) \otimes V_{\alpha, \beta}^{\prime}$ is not simple, we still can get simple modules by considering submodules or subquotients of it.

\section{SimPLICITY}

In this section we will prove Theorem 1. Fix any $c, h, \alpha, \beta \in \mathbb{C}$ with $0 \leq \mathfrak{R e} \alpha<1$ and $\beta \neq 1$. Let $V$ be a highest weight module (not necessarily simple) with highest weight vector $u$ of highest weight $(c, h)$ and $V_{\alpha, \beta}$ have a basis as in (1.1). We will consider the tensor product modules $V \otimes V_{\alpha, \beta}$ and $V \otimes V_{\alpha, \beta}^{\prime}$. Let us first introduce our "shifting technique" for the tensor product.

The vector space $L=V \otimes \mathbb{C}\left[t^{ \pm 1}\right]$ can be endowed with a Vir-module structure via

$$
d_{k}\left(P u \otimes t^{n}\right)=\left(d_{k}+\alpha+n-\operatorname{deg}(P)+k \beta\right) P u \otimes t^{n+k},
$$


for all $k, n \in \mathbb{Z}$ and homogeneous $P \in U\left(\right.$ Vir $\left._{-}\right)$, and the action of $C$ is the scalar $c$. When $\alpha=\beta=0$, we see that $L$ has a submodule spanned by $P u \otimes t^{\operatorname{deg}(P)}$ for all homogeneous $P \in U\left(\mathrm{Vir}_{-}\right)$, which is just a copy of $V(c, h)$; let $L^{\prime}$ be the corresponding quotient module in this case, and we denote $L^{\prime}=L$ otherwise.

It is easy to check that $V \otimes V_{\alpha, \beta} \cong L$ as Virasoro modules via the following map: for any $m \in \mathbb{Z}_{+}$

$V \otimes V_{\alpha, \beta} \rightarrow L, \quad P u \otimes v_{n} \mapsto P u \otimes t^{n+\operatorname{deg}(P)}, \forall n \in \mathbb{Z}, P \in U\left(\mathrm{Vir}_{-}\right)_{-m}$.

Also this map induces an isomorphism $V \otimes V_{\alpha, \beta}^{\prime} \cong L^{\prime}$ in the case $\alpha=$ $\beta=0$. Thus in the following of this section we will consider $L$ and $L^{\prime}$ instead of $V \otimes V_{\alpha, \beta}$ and $V \otimes V_{\alpha, \beta}^{\prime}$. The advantage of the "shifting technique" of the notation for $L=\bigoplus_{n \in \mathbb{Z}} V \otimes t^{n}$ is the weight space decomposition, that is,

$$
V \otimes t^{n}=\left\{x \in L \mid d_{0} x=(\alpha+h+n) x\right\}, \forall n \in \mathbb{Z} .
$$

We will see the power of this advantage in the following proofs.

The following observation is obvious

Lemma 4. The Virasoro module $L^{\prime}$ is generated by $\left\{u \otimes t^{k} \mid k \in \mathbb{Z}\right\}$.

From now on in this section, we assume that $V=V(c, h)$ is the simple highest weight module with highest weight $(c, h)$. Thus $L \cong$ $V(c, h) \otimes V_{\alpha, \beta}$ and $L^{\prime} \cong V(c, h) \otimes V_{\alpha, \beta}^{\prime}$. The following lemma is crucial in this paper.

Lemma 5. For any nonzero Vir-submodule $W$ of $L^{\prime}$, there exists $N \in \mathbb{Z}$ such that $V(c, h) \otimes t^{n} \subseteq W$ for all $n \geq N$.

Proof. Since $L^{\prime}$ is a weight module, there exist subspaces $W_{n} \subseteq V(c, h)$ for all $n \in \mathbb{Z}$ such that $W=\bigoplus_{n \in \mathbb{Z}} W_{n} \otimes t^{n}$. For any nonzero vector $w \in W_{n}$ we can find homogeneous $P_{i} \in U\left(\right.$ Vir $\left._{-}\right)$such that

$$
w=\sum_{i=1}^{r} P_{i} u, \quad \text { where } 0 \geq \operatorname{deg}\left(P_{1}\right)>\operatorname{deg}\left(P_{2}\right)>\cdots>\operatorname{deg}\left(P_{r}\right) .
$$

Choose $w$ with $r$ being minimal among all nonzero elements in all $W_{n}, n \in \mathbb{Z}$. Suppose this $w \in W_{k}$. Denote $l_{i}=-\operatorname{deg}\left(P_{i}\right)$. If $\alpha=\beta=0$ we further assume that $\operatorname{deg}\left(P_{i}\right) \neq k$, i.e., $l_{i} \neq-k$ for all $i=1, \cdots, r$ since we are working within $L^{\prime}$. For $m, n>-\operatorname{deg}\left(P_{r}\right)$, we have

$$
\begin{gathered}
d_{m} d_{n}\left(w \otimes t^{k}\right)=\sum_{i=1}^{r}\left(\alpha+n+k+l_{i}+\beta m\right)\left(\alpha+k+l_{i}+\beta n\right) w_{i} \otimes t^{m+n+k} \\
d_{m+n}\left(w \otimes t^{k}\right)=\sum_{i=1}^{r}\left(\alpha+k+l_{i}+\beta(m+n)\right) w_{i} \otimes t^{m+n+k} .
\end{gathered}
$$


Thus for all $j: 1 \leq j \leq r$ we have

$$
\begin{gathered}
\left(\alpha+k+l_{j}+\beta(m+n)\right) d_{m} d_{n}\left(w \otimes t^{k}\right) \\
-\left(\alpha+n+k+l_{j}+\beta m\right)\left(\alpha+k+l_{j}+\beta n\right) d_{m+n}\left(w \otimes t^{k}\right) \\
=\sum_{i=1}^{r}\left(\left(\alpha+k+l_{j}+\beta(m+n)\right)\left(\alpha+n+k+l_{i}+\beta m\right)\left(\alpha+k+l_{i}+\beta n\right)\right. \\
\left.\quad-\left(\alpha+n+k+l_{j}+\beta m\right)\left(\alpha+k+l_{j}+\beta n\right)\left(\alpha+k+l_{i}+\beta(m+n)\right)\right) \\
\quad \cdot w_{i} \otimes t^{m+n+k} \\
=\sum_{i=1}^{r}\left(l_{i}-l_{j}\right)\left(\beta^{2}\left(m^{2}+m n+n^{2}\right)+\beta m n+\beta\left(2 \alpha+2 k+l_{i}+l_{j}\right)(m+n)\right. \\
\left.+\left(\alpha+k+l_{i}\right)\left(\alpha+k+l_{j}\right)\right) w_{i} \otimes t^{m+n+k} \in W .
\end{gathered}
$$

By the minimality of $r$, we have

$$
\begin{aligned}
\beta^{2}\left(m^{2}+m n\right. & \left.+n^{2}\right)+\beta m n+\beta\left(2 \alpha+2 k+l_{i}+l_{j}\right)(m+n) \\
& +\left(\alpha+k+l_{i}\right)\left(\alpha+k+l_{j}\right)=0,
\end{aligned}
$$

for all $i \neq j$ and $m, n>l_{r}$. If $r>1$, then we can deduce $\beta=\alpha=0$ and $\left(k+l_{i}\right)\left(k+l_{j}\right)=0$, impossible. As a result, we have $r=1$ and $w=P_{1} u \in W_{k}$ such that $\operatorname{deg}\left(P_{1}\right) \neq k$ when $\alpha=\beta=0$.

There exists $N_{1} \in \mathbb{Z}$ such that $d_{n} P_{1} u=0$ and $\alpha+k-\operatorname{deg}\left(P_{1}\right)+\beta n \neq 0$ for any $n \geq N_{1}-k$. Thus we have

$$
d_{n} \cdot\left(P_{1} u \otimes t^{k}\right)=\left(\alpha+k-\operatorname{deg}\left(P_{1}\right)+\beta n\right)\left(P_{1} u \otimes t^{n+k}\right) \in W
$$

and hence $P_{1} u \in W_{m}$ for all $m \geq N_{1}$.

For any $i \in \mathbb{N}$ and $m \geq N_{1}$, we have $P_{1} u \in W_{m+i}$ and

$$
d_{i} \cdot\left(P_{1} u \otimes t^{m}\right)=\left(d_{i}+\alpha+m-\operatorname{deg}\left(P_{1}\right)+\beta i\right)\left(P_{1} u \otimes t^{m+i}\right) \in W,
$$

yielding that $d_{i} P_{1} u \in W_{m+i}$ for all $m \geq N_{1}$. Inductively, we can show $x P_{1} u \in W_{m+\operatorname{deg}(x)}$ for all homogeneous $x \in U\left(\operatorname{Vir}_{+}\right)$and $m \geq N_{1}$. Since $V(c, h)$ is an irreducible Vir-module, we can find some homogeneous $y \in U\left(\operatorname{Vir}_{+}\right)$such that $y P_{1} u=u$ and hence $u \in W_{m}$ for all $m \geq N=$ $N_{1}+\operatorname{deg}(y)$.

For any $i \in \mathbb{N}$ and $m \geq N$, we have

$$
d_{-i} \cdot\left(u \otimes t^{m+i}\right)=\left(d_{-i}+\alpha+m+i-\beta i\right)\left(u \otimes t^{m}\right) \in W
$$

which implies that $d_{-i} u \in W_{m}$ for all $m \geq N$. Proceeding by downward induction on $\operatorname{deg}(P), P \in U\left(\mathrm{Vir}_{-}\right)$we can deduce that $P u \in W_{m}$ for all homogeneous $P \in U$ (Vir V $_{-}$) and $m \geq N$, that is, $W_{m}=V(c, h)$ for all $m \geq N$, as desired.

From the proof of the above lemma we can get the following corollaries. 
Corollary 6. For any $n \in \mathbb{N}$, the submodule generated by all $V(c, h) \otimes$ $t^{k}, k>n$ is the same as the submodule generated by all $u \otimes t^{k}, k>n$. We denote this submodule by $W^{(n)}$.

Corollary 7. $L^{\prime}$ is simple if and only if it is generated by $u \otimes t^{k}$ for some sufficiently large $k \in \mathbb{N}$.

Now we introduce our linear map $\widetilde{\varphi}_{n}: M(c, h) \rightarrow \mathbb{C}$ for $n \in \mathbb{Z}$. Denote by $T\left(\mathrm{Vir}_{-}\right)$the tensor algebra of $\mathrm{Vir}_{-}$, i.e.,

$$
T\left(\operatorname{Vir}_{-}\right)=\mathbb{C} \oplus\left(\operatorname{Vir}_{-}\right) \oplus\left(\operatorname{Vir}_{-} \otimes \operatorname{Vir}_{-}\right) \oplus \cdots\left(\text { Vir }_{-}{ }^{\otimes k}\right) \oplus \cdots
$$

with multiplication being the tensor product, which is a free associative algebra. For any $k \in \mathbb{N}$, define $\operatorname{deg}\left(d_{-k}\right)=-k$ and $\operatorname{deg}(1)=0$, then $T\left(\right.$ Vir $\left._{-}\right)$becomes a $\mathbb{Z}_{-}$-graded algebra. Now for any $n \in \mathbb{Z}$ and $\alpha, \beta \in$ $\mathbb{C}$, we can inductively define a linear map $\varphi_{n}: T\left(\operatorname{Vir}_{-}\right) \rightarrow \mathbb{C}$ as follows:

$$
\varphi_{n}(1)=1, \quad \varphi_{n}\left(d_{-k} P\right)=-(\alpha+n+k-\operatorname{deg}(P)-k \beta) \varphi_{n}(P),
$$

for any homogeneous element $P \in T\left(\mathrm{Vir}_{-}\right)$. Notice that $U\left(\mathrm{Vir}_{-}\right)$is just the quotient $T\left(\mathrm{Vir}_{-}\right) / J$ where $J$ is the two-sided ideal generated by $d_{-i} d_{-j}-d_{-j} d_{-i}-(i-j) d_{-i-j}$ for $i, j \in \mathbb{N}$. Now we will show $J \subseteq \operatorname{ker}\left(\varphi_{n}\right)$. By the definition of $\varphi_{n}$, it is enough to show

$$
\varphi_{n}\left(\left(d_{-i} d_{-j}-d_{-j} d_{-i}-(i-j) d_{-i-j}\right) P\right)=0
$$

for any homogeneous element $P \in T\left(\operatorname{Vir}_{-}\right)$. Let $m=\operatorname{deg}(P)$, we have

$$
\begin{aligned}
& \varphi_{n}\left(\left(d_{-i} d_{-j}-d_{-j} d_{-i}\right) P\right) \\
= & (n-m+j+i+\alpha-i \beta)(n-m+j+\alpha-j \beta) \varphi_{n}(P) \\
& \quad-(n-m+i+j+\alpha-j \beta)(n-m+i+\alpha-i \beta) \varphi_{n}(P) \\
= & (j-i)(n-m+i+j+\alpha-(i+j) \beta) \varphi_{n}(P) \\
= & (i-j) \varphi_{n}\left(d_{-i-j} P\right) .
\end{aligned}
$$

Thus $\varphi_{n}$ induces a linear map on $U\left(\operatorname{Vir}_{-}\right)$, still denoted by $\varphi_{n}$. Since the Verma module $M(c, h)$ is free of rank 1 as a $U\left(\mathrm{Vir}_{-}\right)$-module, we can define the linear map

$$
\widetilde{\varphi}_{n}: M(c, h) \rightarrow \mathbb{C}, \quad \widetilde{\varphi}_{n}(P u)=\varphi_{n}(P), \forall P \in U\left(\operatorname{Vir}_{-}\right) .
$$

Lemma 8. Let $W$ be a Vir-submodule of $L^{\prime}$ with $W \supseteq W^{(n)}$ for some $n \in \mathbb{Z}$. Then

$$
P u \otimes t^{n} \equiv \varphi_{n}(P) u \otimes t^{n} \quad(\bmod W), \forall P \in U\left(\operatorname{Vir}_{-}\right) .
$$

Proof. Let $Q \in U\left(\mathrm{Vir}_{-}\right)$be a homogeneous element and suppose that the result holds for this $Q$. Then for any $k \in \mathbb{N}$, we have

$$
d_{-k}\left(Q u \otimes t^{n+k}\right)=\left(\left(d_{-k}+\alpha+n+k-\operatorname{deg}(Q)-k \beta\right) Q u\right) \otimes t^{n} \in W,
$$


which indicates

$$
\begin{aligned}
\left(d_{-k} Q u\right) \otimes t^{n} & \equiv-(\alpha+n+k-\operatorname{deg}(Q)-k \beta)(Q u) \otimes t^{n} \\
& \equiv-(\alpha+n+k-\operatorname{deg}(Q)-k \beta) \varphi_{n}(Q) u \otimes t^{n} \\
& \equiv \varphi_{n}\left(d_{-k} Q\right) u \otimes t^{n}(\bmod W) .
\end{aligned}
$$

The lemma follows from induction on $\operatorname{deg}(Q)$ and linearity of $\varphi_{n}$.

Let $J(c, h)$ be the maximal submodule of $M(c, h)$. By [FF], we can assume that $J(c, h)$ is generated by two singular vectors $Q_{1}$ and $Q_{2}$ for some homogeneous $Q_{1}, Q_{2} \in U\left(\right.$ Vir $\left._{-}\right)$. We have made the assumption that $Q_{1}=Q_{2}$ if $J(c, h)$ can be generated by a singular vector and $Q_{1}=Q_{2}=0$ if $M(c, h)$ itself is simple. Under this assumption, $Q_{1}$ and $Q_{2}$ are both homogeneous and unique up to nonzero scalars. Now we can give the proof of Theorem 1 .

Proof of Theorem 1. Recall that $\beta \neq 1$. Let $W$ be a nonzero Virsubmodule of $L^{\prime} \cong V(c, h) \otimes V_{\alpha, \beta}^{\prime}$. By Lemma 5 we know that there is $N \in \mathbb{Z}$ such that $V(c, h) \otimes t^{k} \subseteq W$ for all $k>N$. By Lemma 8 we have

$$
Q_{i} u \otimes t^{N}-\varphi_{N}\left(Q_{i}\right) u \otimes t^{N} \in W, \quad i=1,2 .
$$

"If part of (a)." By the hypothesis, we have $\varphi_{N}\left(Q_{1}\right) \neq 0$ or $\varphi_{N}\left(Q_{2}\right) \neq$ 0 , we get $u \otimes t^{N} \in W$. Inductively, we get $u \otimes t^{k} \in W$ for all $k \in \mathbb{Z}$. By Lemma 4, we have $W=L^{\prime}$, that is, $L^{\prime}$ is simple.

"Only if part of (a)." Now we assume that the equations $\varphi_{n}\left(Q_{1}\right)=$ $\varphi_{n}\left(Q_{2}\right)=0$ has at least one solution for $n$ (nonzero if $(\alpha, \beta)=(0,0)$ ).

We first consider the case that $\varphi_{n}\left(Q_{1}\right)=\varphi_{n}\left(Q_{2}\right)=0$ has only finitely many such solutions and prove (b). If $(\alpha, \beta) \neq(0,0)$, let $N_{0}$ be the largest integer such that $\varphi_{n}\left(Q_{1}\right)=\varphi_{n}\left(Q_{2}\right)=0$. Similarly as in the "if part", we get that $u \otimes t^{k} \in W$ for all $k>N_{0}$ by induction. If $(\alpha, \beta)=(0,0)$, let $N_{0}$ be the largest nonzero integer such that $\varphi_{n}\left(Q_{1}\right)=\varphi_{n}\left(Q_{2}\right)=0$. Since $u \otimes t^{0}=0$ is already in $W \subseteq L^{\prime}$, again we can get $u \otimes t^{k} \in W$ for all $k>N_{0}$. Recall that $W^{\left(N_{0}\right)}$ is the Virsubmodule of $L^{\prime}$ generated by all $V(c, h) \otimes t^{k}, k>N_{0}$. In both cases we conclude that $W \supseteq W^{\left(N_{0}\right)}$ from Corollary 6 , implying that $W^{\left(N_{0}\right)}$ is the smallest submodule of $L^{\prime}$ which has to be simple. Since some weight spaces of $W^{\left(N_{0}\right)}$ are infinite-dimensional, from [MZ1] we know that any nonzero weight spaces are infinite-dimensional. Thus (b) follows.

Now we go back to the proof of (a). Let $N$ be any (nonzero if $(\alpha, \beta)=(0,0))$ integer such that $\varphi_{N}\left(Q_{1}\right)=\varphi_{N}\left(Q_{2}\right)=0$. We will show that $W^{(N)}$ is a proper submodule of $L^{\prime}$. By the definition of $\varphi_{N}$ in (2.2), we obtain that

$$
\begin{aligned}
\widetilde{\varphi}_{N}(J(c, h)) & =\widetilde{\varphi}_{N}\left(U\left(\operatorname{Vir}_{-}\right) Q_{1} u+U\left(\operatorname{Vir}_{-}\right) Q_{2} u\right) \\
& =\varphi_{N}\left(U\left(\operatorname{Vir}_{-}\right) Q_{1}\right)+\varphi_{N}\left(U\left(\operatorname{Vir}_{-}\right) Q_{2}\right)=0 .
\end{aligned}
$$


Thus $\widetilde{\varphi}_{N}$ induces a linear map $V(c, h) \rightarrow \mathbb{C}$ which sends $P u$ to $\varphi_{N}(P)$ for any $P \in U\left(\operatorname{Vir}_{-}\right)$. Since $\widetilde{\varphi}_{N}(u)=1$ and hence $\operatorname{ker}\left(\widetilde{\varphi}_{N}\right) \neq V(c, h)$.

First, by the PBW theorem, the weight space of $W^{(N)}$ with weight $\alpha+h+N$ is

$$
\begin{aligned}
W_{N}^{(N)} \otimes t^{N} & =\sum_{k \in \mathbb{N}} d_{-k}\left(V(c, h) \otimes t^{N+k}\right) \\
& =\sum_{k \in \mathbb{N}} \sum_{P \in U\left(\operatorname{Vir}_{-}\right)}\left(\left(d_{-k}+\alpha+N+k-\operatorname{deg}(P)-k \beta\right) P u\right) \otimes t^{N}
\end{aligned}
$$

or in other words,

$$
W_{N}^{(N)}=\sum_{k \in \mathbb{N}} \sum_{P \in U\left(\text { Vir }_{-}\right)}\left(\left(d_{-k}+\alpha+N+k-\operatorname{deg}(P)-k \beta\right) P u\right),
$$

where the second summation is taken over all homogeneous $P$.

On the other hand, for any homogeneous element $P$, we have

$$
\begin{aligned}
& \widetilde{\varphi}_{N}\left(\left(d_{-k}+\alpha+N+k-\operatorname{deg}(P)-k \beta\right) P u\right) \\
= & \varphi_{N}\left(d_{-k} P\right)+(\alpha+N+k-\operatorname{deg}(P)-k \beta) \varphi_{N}(P)=0 .
\end{aligned}
$$

That is $W_{N}^{(N)} \subseteq \operatorname{ker}\left(\widetilde{\varphi}_{N}\right)$, as desired. By Lemma 8 we see that actually $W_{N}^{(N)}=\operatorname{ker}\left(\widetilde{\varphi}_{N}\right)$. Thus $W^{(N)}$ is a proper submodule of $L^{\prime}$, i.e., $L^{\prime}$ is not simple.

Let $\Phi$ be the set of all (nonzero if $(\alpha, \beta)=(0,0))$ integers $n$ such that $\varphi_{n}\left(Q_{1}\right)=\varphi_{n}\left(Q_{2}\right)=0$. From the above theorem, we can have the following comments on the submodules of $L^{\prime}$.

Remark 9. (1) We have a sequence of submodules of $L^{\prime}$ :

$$
\cdots \subseteq W^{(n+1)} \subseteq W^{(n)} \subseteq W^{(n-1)} \subseteq \cdots
$$

If $Q_{1}=Q_{2}=0$, that is, $V(c, h)=M(c, h)$, then $\Phi=\mathbb{Z}$ except that $\Phi=\mathbb{Z} \backslash\{0\}$ when $(\alpha, \beta)=(0,0)$ and all inclusions $W^{(n+1)} \subseteq$ $W^{(n)}$ are proper for $n \in \Phi$. It is easy to see that $W^{(n)} / W^{(n-1)}$ is a highest weight module of highest weight $\alpha+h+n$ for any $n \in \Phi$. In particular $V(c, h) \otimes V_{\alpha, \beta}^{\prime}$ is an extension of countably many highest weight modules.

If $Q_{1} \neq 0$ or $Q_{2} \neq 0$, then $\Phi$ is a finite subset of $\mathbb{Z}$ and the only proper inclusions in (2.4) are $W^{(n)} \subsetneq W^{(n-1)}$ for $n \in \Phi$. If we write $\Phi=\left\{n_{1}, n_{2}, \ldots, n_{r}\right\}$ with $n_{1}<n_{2}<\cdots<n_{r}$ and take any $n_{0}<n_{1}$, then (2.4) can be simplified as:

$$
0 \subsetneq W^{\left(n_{r}\right)} \subsetneq \cdots \subsetneq W^{\left(n_{2}\right)} \subsetneq W^{\left(n_{1}\right)} \subsetneq W^{\left(n_{0}\right)}=L^{\prime} .
$$

We have known that $W^{\left(n_{r}\right)}$ is the unique minimal nonzero proper submodule of $L^{\prime}$. By replacing $N$ with $n_{i}, 1 \leq i \leq r$ in the proof of 
Theorem 1, we see that

$$
W_{n_{i}}^{\left(n_{i-1}\right)}=V(c, h)=W^{\left(n_{i}\right)} \oplus\left(\mathbb{C} u \otimes t^{n_{i}}\right),
$$

and $W^{\left(n_{i-1}\right)}$ is generated by $W^{\left(n_{i}\right)}$ and $u \otimes t^{n_{i}}$. This implies that $W^{\left(n_{i-1}\right)} / W^{\left(n_{i}\right)}$ is a highest weight module with highest weight $\alpha+h+n_{i}$. Then we can obtain that $W^{\left(n_{r}\right)}$ is a simple weight Vir-module with infinitely dimensional weight spaces.

(2) Recall that $V_{\alpha, 0} \cong V_{\alpha, 1}$ for $\alpha \neq 0$ and in this case they correspond to the same module $L^{\prime}$ while different linear map $\varphi_{n}$, to distinguish them we denote by $\varphi_{n}^{(\alpha, 0)}$ and $\varphi_{n}^{(\alpha, 1)}$ respectively. Then for any homogeneous element $P \in U\left(\mathrm{Vir}_{-}\right)$, we have

$$
\varphi_{n}^{(\alpha, 0)}(P)=\frac{\alpha+n-\operatorname{deg}(P)}{\alpha+n} \varphi_{n}^{(\alpha, 1)}(P),
$$

which results in the same $\Phi$ and hence the same sequence of submodules of $L^{\prime}$ in (2.5).

\section{ISOMORPHISM}

In this section we will prove Theorem 2 and compare the tensor products $V(c, h) \otimes V_{\alpha, \beta}^{\prime}$ with other known simple weight modules.

Proof of Theorem 2. Fix any complex numbers $c, h, c_{1}, h_{1}, \alpha, \beta, \alpha_{1}, \beta_{1}$ with $0 \leq \mathfrak{R e} \alpha, \mathfrak{R e} \alpha_{1}<1, \beta \neq 1$ and $\beta_{1} \neq 1$. Let $V$ (resp. $V_{1}$ ) be a highest weight module generated by highest weight vector $u$ (resp. $u_{1}$ ) with highest weight $(c, h)$ (resp. $\left.\left(c_{1}, h_{1}\right)\right)$. As we did in Section 2, we will identify $V \otimes V_{\alpha, \beta}$ and $V_{1} \otimes V_{\alpha_{1}, \beta_{1}}$ with $L=V \otimes \mathbb{C}\left[t^{ \pm 1}\right]$ and $L_{1}=V_{1} \otimes \mathbb{C}\left[t^{ \pm 1}\right]$ respectively via (2.1) and we also identify $V \otimes V_{\alpha, \beta}^{\prime}$ and $V_{1} \otimes V_{\alpha_{1}, \beta_{1}}^{\prime}$ with $L^{\prime}$ and $L_{1}^{\prime}$ respectively.

The "if part" is trivial. We now prove the "only if part". It is clear that $c=c_{1}$.

Now assume $\psi: L^{\prime} \longrightarrow L_{1}^{\prime}$ is an isomorphism of Vir-modules. Fix any $k \in \mathbb{Z}$ such that $k \neq 0$ when $(\alpha, \beta)=(0,0)$. Since $\psi\left(u \otimes t^{k}\right)$ and $u \otimes t^{k}$ are of the same weight, so $\psi\left(u \otimes t^{k}\right)=w \otimes t^{l}$ for some nonzero vector $w=\sum_{i=0}^{s} w_{i}$ with $w_{i} \in \mathbb{C} P_{i} u_{1}, \operatorname{deg}\left(P_{i}\right)=-i$ and $\alpha+h+k=$ $\alpha_{1}+h_{1}+l$. We may assume that $\alpha_{1}+l+i \neq 0$ if $\beta_{1}=0$. For any $m, n \geq s+1$, we have

$$
\begin{aligned}
& \psi\left(d_{m} d_{n}\left(u \otimes t^{k}\right)\right)=(\alpha+k+n+\beta m)(\alpha+k+\beta n) \psi\left(u \otimes t^{m+n+k}\right) \\
= & d_{m} d_{n}\left(w \otimes t^{l}\right) \\
= & \sum_{i=0}^{s}\left(\alpha_{1}+l+i+n+\beta_{1} m\right)\left(\alpha_{1}+l+i+\beta_{1} n\right) w_{i} \otimes t^{m+n+l}
\end{aligned}
$$


and

$$
\begin{gathered}
\\
\psi\left(d_{m+n}\left(u \otimes t^{k}\right)\right)=(\alpha+k+\beta(m+n)) \psi\left(u \otimes t^{m+n+k}\right) \\
=d_{m+n}\left(w \otimes t^{l}\right)=\sum_{i=0}^{s}\left(\alpha_{1}+l+i+\beta_{1}(m+n)\right) w_{i} \otimes t^{m+n+l} .
\end{gathered}
$$

This implies

$$
\begin{aligned}
& (\alpha+k+\beta(m+n))\left(\alpha_{1}+l+i+n+\beta_{1} m\right)\left(\alpha_{1}+l+i+\beta_{1} n\right) \\
= & \left(\alpha_{1}+l+i+\beta_{1}(m+n)\right)(\alpha+k+n+\beta m)(\alpha+k+\beta n),
\end{aligned}
$$

for all $m, n \geq s+1$ and $0 \leq i \leq s$ with $w_{i} \neq 0$. Rewrite it as a polynomial of $m$ and $n$ as follows

$$
\begin{aligned}
& \beta \beta_{1}\left(\beta_{1}-\beta\right) m n(m+n)+\beta \beta_{1}\left(\alpha_{1}+l+i-\alpha-k\right)\left(m^{2}+n^{2}\right) \\
+ & \left((\alpha+k) \beta_{1}\left(\beta_{1}-2 \beta-1\right)+\left(\alpha_{1}+l+i\right) \beta\left(1+2 \beta_{1}-\beta\right)\right) m n \\
+ & \left((\alpha+k)\left(\alpha_{1}+l+i\right)\left(\beta_{1}-\beta\right)+\beta\left(\alpha_{1}+l+i\right)^{2}-\beta_{1}(\alpha+k)^{2}\right)(m+n) \\
+ & (\alpha+k)\left(\alpha_{1}+l+i\right)\left(\alpha_{1}+l+i-\alpha-k\right)=0,
\end{aligned}
$$

which holds for all $i$ with $w_{i} \neq 0$ and $m, n>s$. Hence we get

(1). $(\alpha+k)\left(\alpha_{1}+l+i\right)\left(\alpha_{1}+l+i-\alpha-k\right)=0$,

(2). $\left(\beta\left(\alpha_{1}+l+i\right)+\beta_{1}(\alpha+k)\right)\left(\alpha_{1}+l+i-\alpha-k\right)=0$,

(3). $\beta \beta_{1}\left(\alpha_{1}+l+i-\alpha-k\right)=0$,

(4). $(\alpha+k) \beta_{1}\left(\beta_{1}-1\right)=\left(\alpha_{1}+l+i\right) \beta(\beta-1)$,

(5). $\beta \beta_{1}\left(\beta_{1}-\beta\right)=0$.

If $\beta=0$ or $\beta_{1}=0$, from the above formulas and the assumption that $0 \leq \mathfrak{R e} \alpha, \mathfrak{R e} \alpha_{1}<1, \beta \neq 1$ and $\beta_{1} \neq 1$ we can easily deduce that $\beta=\beta_{1}=0$ and $\alpha=\alpha_{1}$. Next we assume that $\beta \beta_{1} \neq 0$. Again, from the above formulas and the assumption that $0 \leq \mathfrak{R e} \alpha, \mathfrak{R e} \alpha_{1}<1$, $\beta \neq 1$ and $\beta_{1} \neq 1$ we can easily deduce that $\beta=\beta_{1}$ and $\alpha=\alpha_{1}$. In all cases we can deduce that $k=l+i$ for all $w_{i} \neq 0$, which implies that there is only one integer, say $r$ such that $w_{r} \neq 0$. Now we get $\psi\left(u \otimes t^{k}\right)=P_{r} u_{1} \otimes t^{k-r}$, where $r=h_{1}-h$ since $\alpha+h+k=\alpha_{1}+h_{1}+l$. We will replace $P_{r}$ with $P_{r, k}$ since it also depends on $k$. Note that

$$
\sum_{k \in \mathbb{Z}} U\left(\operatorname{Vir}_{-}\right)\left(u \otimes t^{k}\right)=V \otimes V_{\alpha, \beta}^{\prime} \text { for }(\alpha, \beta) \neq(0,0)
$$

and

$$
\sum_{k \in \mathbb{Z} \backslash\{0\}} U\left(\operatorname{Vir}_{-}\right)\left(u \otimes t^{k}\right)=V \otimes V_{\alpha, \beta}^{\prime} \text { for }(\alpha, \beta)=(0,0),
$$

which together with the fact that $\psi$ is an isomorphism implies that

$$
\sum_{k \in \mathbb{Z}} U\left(\operatorname{Vir}_{-}\right)\left(P_{r, k} u_{1} \otimes t^{k-r}\right)=V_{1} \otimes V_{\alpha_{1}, \beta_{1}}^{\prime} \text { for }(\alpha, \beta) \neq(0,0)
$$


and

$$
\sum_{k \in \mathbb{Z} \backslash\{0\}} U\left(\operatorname{Vir}_{-}\right)\left(P_{r, k} u_{1} \otimes t^{k-r}\right)=V_{1} \otimes V_{\alpha_{1}, \beta_{1}}^{\prime} \text { for }(\alpha, \beta)=(0,0) .
$$

The above equalities force that $P_{r, k} u_{1}$ is a nonzero scalar multiple of $u_{1}$. Thus $r=0$, that is, $h=h_{1}$ and there exist $c_{k} \in \mathbb{C}^{*}$ such that $\psi\left(u \otimes t^{k}\right)=c_{k} u_{1} \otimes t^{k}$ for all $k \in \mathbb{Z}$ but not zero if $(\alpha, \beta)=(0,0)$.

For any $n \in \mathbb{N}$, we have

$$
\begin{aligned}
c_{n+k}(\alpha+k+\beta n) u_{1} \otimes t^{n+k} & =\psi\left((\alpha+k+\beta n) u \otimes t^{n+k}\right)=\psi\left(d_{n}\left(u \otimes t^{k}\right)\right) \\
& =d_{n}\left(\psi\left(u \otimes t^{k}\right)\right)=c_{k} d_{n}\left(u_{1} \otimes t^{k}\right) \\
& =c_{k}(\alpha+k+\beta n) u_{1} \otimes t^{n+k} .
\end{aligned}
$$

If $\alpha+k+\beta n \neq 0$, we get $c_{n+k}=c_{k}$. Now for any (nonzero if $(\alpha, \beta)=$ $(0,0)) k, l \in \mathbb{Z}$, choosing a sufficiently large $n \in \mathbb{N}$ such that

$$
n+k \neq 0, \alpha+k+\beta n \neq 0 \text { and } \alpha+l+\beta(n-l+k) \neq 0,
$$

then we have $c_{k}=c_{n+k}$ and $c_{l}=c_{(n-l+k)+l}=c_{n+k}=a$ for some $a \in \mathbb{C}^{*}$. By replacing $\psi$ with its multiple we may assume that $\psi\left(u \otimes t^{k}\right)=u_{1} \otimes t^{k}$ for all $k \in \mathbb{Z}$ but not zero if $(\alpha, \beta)=(0,0)$. Suppose $X$ be the maximal subspace of $U\left(\right.$ Vir $\left._{-}\right)$such that

$$
\psi\left((P u) \otimes t^{k}\right)=\left(P u_{1}\right) \otimes t^{k}, \forall P \in X, \forall k \in \mathbb{Z} .
$$

We can easily see that $X=U\left(\right.$ Vir $\left._{-}\right)$and the map $V \rightarrow V_{1}$ defined by $P u \rightarrow P u^{\prime}$ is a module isomorphism. So $V \cong V_{1}$.

Now we compare the tensor products $V(c, h) \otimes V_{\alpha, \beta}^{\prime}$ with other known simple weight modules. So far known simple weight Virasoro modules are from [CM, LLZ]. It is proved in [LLZ] that $V(c, h) \otimes V_{\alpha, \beta}^{\prime}$ (or its minimal submodule) is not isomorphic to any module in [LLZ]. Next we compare $V(c, h) \otimes V_{\alpha, \beta}^{\prime}$ with simple weight modules from [CM].

Theorem 10. Let $c, h, \alpha, \beta, a, b, \gamma, p \in \mathbb{C}$. Then $V(c, h) \otimes V_{\alpha, \beta}^{\prime}$ (or its minimal submodule) is not isomorphic to any irreducible module $E_{a}(b, \gamma, p)$ defined in $\mathrm{CM}$.

Proof. Let us recall the Casimir operators $Q_{n}=d_{0}^{2}+n d_{0}-d_{-n} d_{n} \in$ $U$ (Vir) for any $n \in \mathbb{N}$. From Page 174 on [CM], we know that for any $w \in E_{a}(b, \gamma, p)$,

$$
\operatorname{dim} \operatorname{span}\left\{Q_{n} w \mid n \in \mathbb{N}\right\}<\infty .
$$

At the same time, in $V(c, h) \otimes V_{\alpha, \beta}^{\prime}$ (or its minimal submodule) we have

$$
\operatorname{dim} \operatorname{span}\left\{Q_{n}\left(u \otimes v_{k}\right) \mid n \in \mathbb{N}\right\}=\infty,
$$

for any $k \in \mathbb{Z}$ with $v_{k} \neq 0$. The theorem follows. 


\section{EXAmples}

In this section, to illustrate our Theorem 1 we will present some examples for special values of $c, h$ and $\alpha, \beta$. Notations are as in Section 2. To distinguish different modules relative to different $\alpha, \beta$ we will add subscripts for certain modules, such as $W_{\alpha, \beta}^{(n)}$ and so on, if necessary. Recall that $0 \leq \Re(\alpha)<1$ and $\beta \neq 1$.

Example 11. We first give some examples such that the unique maximal submodule $J(c, h)$ of the corresponding Verma module $M(c, h)$ can be generated by only one singular vector. We point out that the expression for $h$ at the very beginning in Sect.5 in $[\mathrm{A}]$ should be $h=\frac{m^{2}-(p+q)^{2}}{4 p q}$.

(I). Let $(c, h)=(1,0)$. In this case we take $p=-q=1$ and $m=0$ in Sect.5 of [A]. From Theorem A in [A] we get that $J(1,0)=U\left(\operatorname{Vir}_{-}\right) Q u$, where $Q=d_{-1}$. It is clear that $\varphi_{n}(Q)=(\beta-\alpha-n-1)$. From Theorem 1 we see that $V(1,0) \otimes V_{\alpha, \beta}^{\prime}$ is simple if and only if $\beta-\alpha \notin \mathbb{Z}$. Moreover, if $\beta-\alpha \in \mathbb{Z}$, then $V(1,0) \otimes V_{\alpha, \beta}^{\prime}$ has a unique simple submodule $W_{\alpha, \beta}^{(\beta-\alpha-1)}$ and $\left(V(1,0) \otimes V_{\alpha, \beta}^{\prime}\right) / W_{\alpha, \beta}^{(\beta-\alpha-1)}$ is a highest weight module of highest weight $(1, \beta-1)$. It is easy to see that similar result holds for any module $V(c, 0)$ provided that the corresponding maximal submodule $J(c, 0)$ is generated by $d_{-1} u$ (see Example 14).

(II). Let $(c, h)=\left(1,-\frac{1}{4}\right)$. In this case we take $p=-q=1$ and $m=1$ in Sect.5 of [A]. Then we see that $J\left(1,-\frac{1}{4}\right)$ is generated by only one singular vector of degree 2 . By direct computation we can deduce that $J\left(1,-\frac{1}{4}\right)=U\left(\right.$ Vir $\left._{-}\right) Q u$ where $Q=d_{-1}^{2}+d_{-2}$. We have

$$
\varphi_{n}(Q)=(\beta-\alpha-n-2)(\beta-\alpha-n-1)+(2 \beta-\alpha-n-2) .
$$

Solving the equation $\varphi_{n}(Q)=0$ for $n$, we get

$$
n_{1}=\beta-\alpha-1+\sqrt{1-\beta}, n_{2}=\beta-\alpha-1-\sqrt{1-\beta} .
$$

(1). $V\left(1,-\frac{1}{4}\right) \otimes V_{\alpha, \beta}^{\prime}$ is simple if $n_{1}, n_{2} \notin \mathbb{Z}$.

(2). If for some $\alpha, \beta$, the numbers $n_{1} \in \mathbb{Z}$ (resp. $n_{2} \in \mathbb{Z}$ ) and $n_{2} \notin \mathbb{Z}$ (resp. $\left.\quad n_{1} \notin \mathbb{Z}\right)$, then $V\left(1,-\frac{1}{4}\right) \otimes V_{\alpha, \beta}^{\prime}$ has a unique simple submodule $W_{\alpha, \beta}^{\left(n_{1}\right)}$ (resp. $\left.W_{\alpha, \beta}^{\left(n_{2}\right)}\right)$. Moreover, $\left(V\left(1,-\frac{1}{4}\right) \otimes V_{\alpha, \beta}^{\prime}\right) / W_{\alpha, \beta}^{\left(n_{1}\right)}$ (resp. $\left.\left(V\left(1,-\frac{1}{4}\right) \otimes V_{\alpha, \beta}^{\prime}\right) / W_{\alpha, \beta}^{\left(n_{2}\right)}\right)$ is a highest weight module of highest weight $\left(1, \beta-\frac{5}{4}+\sqrt{1-\beta}\right)\left(\operatorname{resp} .\left(1, \beta-\frac{5}{4}-\sqrt{1-\beta}\right)\right.$ ).

(3). If $n_{1}, n_{2} \in \mathbb{Z}$, then we have two cases

(i). $\alpha=0, \beta=1-k^{2}, n_{1}=-k^{2}+k$ and $n_{2}=-k^{2}-k$ for $k \in \mathbb{N}$;

(ii). $\alpha=\frac{1}{4}, \beta=1-\left(l+\frac{1}{2}\right)^{2}, n_{1}=-l^{2}$ and $n_{2}=-(l+1)^{2}$ for $l \in \mathbb{Z}_{+}$.

In case $\alpha=0$ and $\beta=0$, the module $V\left(1,-\frac{1}{4}\right) \otimes V_{0,0}^{\prime}$ has a unique simple submodule $W_{0,0}^{(-2)}$ and $\left(V\left(1,-\frac{1}{4}\right) \otimes V_{0,0}^{\prime}\right) / W_{0,0}^{(-2)}$ is a highest weight 
module of highest weight $\left(1,-\frac{9}{4}\right)$. In the rest cases, $V\left(1,-\frac{1}{4}\right) \otimes V_{\alpha, \beta}^{\prime}$ has a unique simple submodule $W_{\alpha, \beta}^{\left(n_{1}\right)}$ and another proper submodule $W_{\alpha, \beta}^{\left(n_{2}\right)}$ strictly containing $W_{\alpha, \beta}^{\left(n_{1}\right)}$; moreover, $W_{\alpha, \beta}^{\left(n_{2}\right)} / W_{\alpha, \beta}^{\left(n_{1}\right)}$ is a highest weight module of highest weight $\left(1, n_{1}+\alpha-\frac{1}{4}\right)$ and $\left(V\left(1,-\frac{1}{4}\right) \otimes V_{\alpha, \beta}^{\prime}\right) / W_{\alpha, \beta}^{\left(n_{2}\right)}$ is a highest weight module of highest weight $\left(1, n_{2}+\alpha-\frac{1}{4}\right)$.

(III). Let $(c, h)=(1,-1)$. In this case we take $p=-q=1$ and $m=2$. Then from $[\mathrm{FF}]$ we see that $J(1,-1)$ is generated by only one singular vector of degree 3 . By direct computation we can deduce that $J(1,-1)=U\left(\right.$ Vir $\left._{-}\right) Q u$. where $Q=d_{-1}^{3}+4 d_{-2} d_{-1}+2 d_{-3}$. We deduce that

$$
\begin{aligned}
\varphi_{n}(Q)=(\beta-\alpha-n-3)(\beta-\alpha-n-2)(\beta-\alpha-n-1) \\
\quad+4(2 \beta-\alpha-n-3)(\beta-\alpha-n-1)+2(3 \beta-\alpha-n-3) \\
=(\beta-\alpha-n)\left(n^{2}+2(1-\beta+\alpha) n+2 \alpha-2 \beta \alpha+\beta^{2}-3+\alpha^{2}+2 \beta\right) .
\end{aligned}
$$

Here, we just consider the case that all roots of the equation $\varphi_{n}(Q)=0$ for $n$ are integers. It is not hard to see that we have two cases:

(1). $\alpha=0, \beta=1-k^{2}, n_{1}=-k^{2}+2 k, n_{2}=-k^{2}+1$ and $n_{3}=$ $-k^{2}-2 k$ for $k \in \mathbb{N}$;

(2). $\alpha=\frac{3}{4}, \beta=1-\left(l+\frac{1}{2}\right)^{2}, n_{1}=-l^{2}+l, n_{2}=-l^{2}-l$ and $n_{3}=-l^{2}-3 l-2$ for $l \in \mathbb{Z}_{+}$.

In case $\alpha=0$ and $\beta=0$, the module $V(1,-1) \otimes V_{0,0}^{\prime}$ has different proper submodules $W_{0,0}^{(-3)}$ and $W_{0,0}^{(1)}$ where $W_{0,0}^{(1)}$ is simple. We know that the quotients $W_{0,0}^{(-3)} / W_{0,0}^{(1)}$ and $\left(V(1,-1) \otimes V_{0,0}^{\prime}\right) / W_{0,0}^{(-3)}$ are highest weight modules. In the remaining cases, $V(1,-1) \otimes V_{\alpha, \beta}^{\prime}$ has three different proper submodules $W_{\alpha, \beta}^{\left(n_{i}\right)}$ where $i=1,2,3$.

Example 12. Now we give some examples such that the unique maximal submodule $J(c, h)$ of the Verma module $M(c, h)$ cannot be generated by one singular vector.

(I). Let $(c, h)=(0,0)$. We have $J(0,0)=U\left(\right.$ Vir $\left._{-}\right) Q_{1} u+U\left(\right.$ Vir $\left._{-}\right) Q_{2} u$, where $Q_{1}=d_{-1}$ and $Q_{2}=d_{-2}$. Then $\varphi_{n}\left(Q_{1}\right)=(\beta-\alpha-n-1)$ and $\varphi_{n}\left(Q_{2}\right)=(2 \beta-\alpha-n-2)$. From $\varphi_{n}\left(Q_{1}\right)=\varphi_{n}\left(Q_{2}\right)=0$ we deduce $\alpha=0, \beta=1$. From the assumption that $\beta \neq 1$, we do not have any solutions for $n$ with $\varphi_{n}\left(Q_{1}\right)=\varphi_{n}\left(Q_{2}\right)=0$. Then $V(0,0) \otimes V_{\alpha, \beta}^{\prime}$ is always simple. Indeed, we have $V(0,0) \otimes V_{\alpha, \beta}^{\prime} \cong V_{\alpha, \beta}^{\prime}$.

(II). Let $(c, h)=\left(-\frac{22}{5}, 0\right)$. In this case we take $p=2, q=-5$ and $m=3$. Then from [FF] we see that $J(c, h)$ is generated by two singular vectors of degree 1 and 4 respectively. It is not hard to verify that $J(c, h)=U\left(\operatorname{Vir}_{-}\right) Q_{1} u+U\left(\operatorname{Vir}_{-}\right) Q_{2} u$, where $Q_{1}=d_{-1}$ and $Q_{2}=$ $3 d_{-2}^{2}+5 d_{-4}$. Then we can get

$$
\varphi_{n}\left(Q_{1}\right)=\beta-\alpha-n-1
$$


and

$$
\varphi_{n}\left(Q_{2}\right)=3(2 \beta-\alpha-n-4)(2 \beta-\alpha-n-2)+5(4 \beta-\alpha-n-4) .
$$

Plugging $n=\beta-\alpha-1$ in the second equation, we have

$$
\varphi_{n}\left(Q_{2}\right)=3(\beta-3)(\beta-1)+5(3 \beta-3)=3(\beta-1)(\beta+2) .
$$

Since $\beta \neq 1$, then $V\left(-\frac{22}{5}, 0\right) \otimes V_{\alpha, \beta}^{\prime}$ is simple if and only if $(\alpha, \beta) \neq$ $(0,-2)$. Moreover, $V\left(-\frac{22}{5}, 0\right) \otimes V_{0,-2}^{\prime}$ has a unique simple submodule $W_{0,-2}^{(-3)}$, and $\left(V\left(-\frac{22}{5}, 0\right) \otimes V_{0,-2}^{\prime}\right) / W_{0,-2}^{(-3)}$ is a highest weight module of highest weight $\left(-\frac{22}{5},-3\right)$ which is an irreducible Verma module.

(III). Let $(c, h)=\left(\frac{1}{2},-\frac{1}{2}\right)$. In this case we take $p=3, q=-4$ and $m=5$. From $[\mathrm{FF}]$ we see that $J(c, h)$ is generated by two singular vectors of degree 2,3 . It is not hard to verify that $J(c, h)=U\left(\mathrm{Vir}_{-}\right) Q_{1} u+$ $U\left(\right.$ Vir $\left._{-}\right) Q_{2} u$, where $Q_{1}=3 d_{-1}^{2}+4 d_{-2}, Q_{2}=4 d_{-1}^{3}+12 d_{-2} d_{-1}+3 d_{-3}$, and $Q_{1} u$ is a singular vector while $Q_{2} u$ is obtained by subtracting some element in $U\left(\right.$ Vir $\left._{-}\right) Q_{1} u$ from the other singular vector. Then

$$
\begin{gathered}
\varphi_{n}\left(Q_{1}\right)=3(\beta-\alpha-n-2)(\beta-\alpha-n-1)+4(2 \beta-\alpha-n-2), \\
\varphi_{n}\left(Q_{2}\right)=4(\beta-\alpha-n-3)(\beta-\alpha-n-2)(\beta-\alpha-n-1) \\
\quad+12(2 \beta-\alpha-n-3)(\beta-\alpha-n-1)+3(3 \beta-\alpha-n-3) .
\end{gathered}
$$

Setting $x=n+\alpha-\beta+2$, the above equations become

$$
f(x)=\varphi_{n}\left(Q_{1}\right)=3 x(x-1)-4(x-\beta)
$$

$g(x)=\varphi_{n}\left(Q_{2}\right)=-4 x(x-1)(x+1)+12(x-\beta+1)(x-1)-3(x-2 \beta+1)$.

Now we get $g(x)=q(x) f(x)+r(x)$, where

$$
q(x)=-\frac{4}{3} x+\frac{8}{9} \quad \text { and } \quad r(x)=-\frac{5}{9}(12 \beta-13) x-15+\frac{130}{9} \beta .
$$

It is easy to see that $12 \beta-13 \neq 0$. Then

$$
\begin{aligned}
f(x)= & \left(-\frac{27 x}{5(12 \beta-13)}+\frac{18(3 \beta-5)}{5(12 \beta-13)^{2}}\right) r(x) \\
& +\frac{18(16 \beta-15)(\beta-1)(2 \beta-1)}{(12 \beta-13)^{2}} .
\end{aligned}
$$

Thus to obtain that $\varphi_{n}\left(Q_{1}\right)=\varphi_{n}\left(Q_{2}\right)=0$, we must have $\beta=\alpha=\frac{1}{2}$ and $n=0$, or $\beta=\frac{15}{16}, \alpha=\frac{7}{16}$ and $n=0$. We have $V\left(\frac{1}{2},-\frac{1}{2}\right) \otimes V_{\alpha, \beta}^{\prime}$ is simple if and only if $(\alpha, \beta) \neq\left(\frac{1}{2}, \frac{1}{2}\right)$ or $\left(\frac{7}{16}, \frac{15}{16}\right)$. Moreover, $V\left(\frac{1}{2},-\frac{1}{2}\right) \otimes$ $V_{\frac{1}{2}, \frac{1}{2}}^{\prime}$ has a unique simple submodule $W_{\frac{1}{2}, \frac{1}{2}}^{(0)}$ and $\left(V\left(\frac{1}{2},-\frac{1}{2}\right) \otimes V_{\frac{1}{2}, \frac{1}{2}}^{\prime}\right) / W_{\frac{1}{2}, \frac{1}{2}}^{(0)}$ is a highest weight module of highest weight $\left(\frac{1}{2}, 0\right) ; V\left(\frac{1}{2},-\frac{1}{2}\right) \otimes V_{\frac{7}{16}}^{\prime}, \frac{15}{16}$ has a unique simple submodule $W_{\frac{7}{16}, \frac{15}{16}}^{(0)}$ and $\left(V\left(\frac{1}{2},-\frac{1}{2}\right) \otimes V_{\frac{7}{16}, \frac{15}{16}}^{\prime}\right) / W_{\frac{7}{16}, \frac{15}{16}}^{(0)}$ is a highest weight module of highest weight $\left(\frac{1}{2},-\frac{1}{16}\right)$. 
(IV). Let $(c, h)=\left(\frac{1}{2}, 0\right)$. In this case we take $p=3, q=-4$ and $m=1$. From [FF] we see that $J(c, h)$ is generated by two singular vectors of degree 1 and 6 . It is not hard to verify that $J(c, h)=$ $U\left(\right.$ Vir $\left._{-}\right) Q_{1} u+U\left(\right.$ Vir $\left._{-}\right) Q_{2} u$, where $Q_{1}=d_{-1}, Q_{2}=64 d_{-2}^{3}-93 d_{-3}^{2}+$ $264 d_{-4} d_{-2}-108 d_{-6}$ and $Q_{1} u$ is a singular vector in $M\left(\frac{1}{2}, 0\right)$ and $Q_{2} u$ is obtained by subtracting some element in $U\left(\mathrm{Vir}_{-}\right) Q_{1} u$ from the other singular vector. Then we can get

$$
\begin{gathered}
\varphi_{n}\left(Q_{1}\right)=\beta-\alpha-n-1, \\
\varphi_{n}\left(Q_{2}\right)=64(2 \beta-\alpha-n-6)(2 \beta-\alpha-n-4)(2 \beta-\alpha-n-2) \\
-93(3 \beta-\alpha-n-6)(3 \beta-\alpha-n-3) \\
+264(4 \beta-\alpha-n-6)(2 \beta-\alpha-n-2) \\
-108(6 \beta-\alpha-n-6) .
\end{gathered}
$$

Substituting $n=\beta-\alpha-1$ in the second equation, we have

$$
\varphi_{n}\left(Q_{2}\right)=2(16 \beta-15)(\beta-1)(2 \beta-1) .
$$

So letting $\varphi_{n}\left(Q_{1}\right)=\varphi_{n}\left(Q_{2}\right)=0$, we have $\beta=\alpha=\frac{1}{2}$ and $n=-1$ or $\beta=\alpha=\frac{15}{16}$ and $n=-1$.

We have $V\left(\frac{1}{2}, 0\right) \otimes V_{\alpha, \beta}^{\prime}$ is simple if and only if $(\alpha, \beta) \neq\left(\frac{1}{2}, \frac{1}{2}\right)$ or $\left(\frac{15}{16}, \frac{15}{16}\right)$. Moreover, $V\left(\frac{1}{2}, 0\right) \otimes V_{\frac{1}{2}, \frac{1}{2}}^{\prime}$ has a unique simple submodule $W_{\frac{1}{2}, \frac{1}{2}}^{(-1)}$ and $\left(V\left(\frac{1}{2}, 0\right) \otimes V_{\frac{1}{2}, \frac{1}{2}}^{\prime}\right) / W_{\frac{1}{2}, \frac{1}{2}}^{(-1)}$ is a highest weight module of highest weight $\left(\frac{1}{2},-\frac{1}{2}\right) ; V\left(\frac{1}{2}, 0\right) \otimes V_{\frac{15}{16}, \frac{15}{16}}^{\prime}$ has a unique simple submodule $W_{\frac{15}{16}, \frac{5}{16}}^{(-1)}$ and $\left(V\left(\frac{1}{2}, 0\right) \otimes V_{\frac{15}{16}, \frac{15}{16}}^{\prime}\right) / W_{\frac{15}{16}, \frac{5}{16}}^{(-1)}$ is a highest weight module of highest weight $\left(\frac{1}{2},-\frac{1}{16}\right)$.

$(\mathrm{V})$. Let $(c, h)=\left(\frac{1}{2},-\frac{1}{16}\right)$. In this case we take $p=3, q=-4$ and $m=2$. From [FF we see that $J(c, h)$ is generated by two singular vectors of degree 2,4 . It is not hard to verify that $J(c, h)=$ $U\left(\right.$ Vir $\left._{-}\right) Q_{1} u+U\left(\right.$ Vir $\left._{-}\right) Q_{2} u$, where $Q_{1}=4 d_{-1}^{2}+3 d_{-2}$ and $Q_{2}=144 d_{-1}^{4}+$ $600 d_{-2} d_{-1}^{2}+264 d_{-3} d_{-1}+49 d_{-2}^{2}+36 d_{-4}$. Then we can get

$$
\begin{aligned}
& \varphi_{n}\left(Q_{1}\right)=4(\beta-\alpha-n-2)(\beta-\alpha-n-1)+3(2 \beta-\alpha-n-2) \\
& \varphi_{n}\left(Q_{2}\right) \\
&=144(\beta-\alpha-n-4)(\beta-\alpha-n-3)(\beta-\alpha-n-2)(\beta-\alpha-n-1) \\
& \quad+600(2 \beta-\alpha-n-4)(\beta-\alpha-n-2)(\beta-\alpha-n-1) \\
& \quad+264(3 \beta-\alpha-n-4)(\beta-\alpha-n-1) \\
& \quad+49(2 \beta-\alpha-n-4)(2 \beta-\alpha-n-2) \\
& \quad+36(4 \beta-\alpha-n-4) .
\end{aligned}
$$

By straightforward computations we deduce that

$$
\varphi_{n}\left(Q_{2}\right)=\varphi_{n}\left(Q_{1}\right) q(n)-20(\alpha+n)(16 \beta-15)
$$


for some polynomial $q(n)$ and

$$
\varphi_{n}\left(Q_{1}\right)=(4 \alpha-8 \beta+9+4 n)(\alpha+n)+2(2 \beta-1)(\beta-1) .
$$

Letting $\varphi_{n}\left(Q_{1}\right)=\varphi_{n}\left(Q_{2}\right)=0$, we get that $\alpha=0$ or $\beta=\frac{15}{16}$. If $\alpha=0$, we deduce that $\beta=\frac{1}{2}$ and $n=0$. If $\beta=\frac{15}{16}$, we can deduce that $\alpha=\frac{1}{16}$ and $n=0$, or $\alpha=\frac{9}{16}$ and $n=-1$. We have $V\left(\frac{1}{2},-\frac{1}{16}\right) \otimes V_{\alpha, \beta}^{\prime}$ is simple if and only if $(\alpha, \beta) \neq\left(0, \frac{1}{2}\right),\left(\frac{1}{16}, \frac{15}{16}\right)$ or $\left(\frac{9}{16}, \frac{15}{16}\right)$.

Moreover, $V\left(\frac{1}{2},-\frac{1}{16}\right) \otimes V_{0, \frac{1}{2}}^{\prime}$ has a unique simple submodule $W_{0, \frac{1}{2}}^{(0)}$ and $\left(V\left(\frac{1}{2},-\frac{1}{16}\right) \otimes V_{0, \frac{1}{2}}^{\prime}\right) / W_{0, \frac{1}{2}}^{(0)}$ is a highest weight module of highest weight $\left(\frac{1}{2},-\frac{1}{16}\right) ; V\left(\frac{1}{2},-\frac{1}{16}\right) \otimes V_{\frac{1}{16}}^{\prime}, \frac{15}{16}$ has a unique simple submodule $W_{\frac{1}{16}, \frac{15}{16}}$ and $\left(V\left(\frac{1}{2},-\frac{1}{16}\right) \otimes V_{\frac{1}{16}}^{\prime}, \frac{15}{16}\right) / W_{\frac{1}{16}, \frac{15}{16}}$ is a highest weight module of highest weight $\left(\frac{1}{2}, 0\right) ; V\left(\frac{1}{2},-\frac{1}{16}\right) \otimes V_{\frac{9}{16}}^{\prime}, \frac{15}{16}$ has a unique simple submodule $W_{\frac{9}{16}}, \frac{15}{16}$ and $\left(V\left(\frac{1}{2},-\frac{1}{16}\right) \otimes V_{\frac{9}{16}}^{\prime}, \frac{15}{16}\right) / W_{\frac{9}{16}}, \frac{15}{16}$ is a highest weight module of highest weight $\left(\frac{1}{2},-\frac{1}{2}\right)$.

Remark 13. Comparing the case (III, IV, V) in the above example, we see that using the tensor product $V\left(\frac{1}{2},-\frac{1}{2}\right) \otimes V_{\alpha, \beta}^{\prime}$ we can and only can get the highest weight modules with highest weight $\left(\frac{1}{2}, 0\right)$ and $\left(\frac{1}{2},-\frac{1}{16}\right)$, using the tensor product $V\left(\frac{1}{2}, 0\right) \otimes V_{\alpha, \beta}^{\prime}$ we can and only can get the highest weight modules with highest weight $\left(\frac{1}{2},-\frac{1}{2}\right)$ and $\left(\frac{1}{2},-\frac{1}{16}\right)$, and using the tensor product $V\left(\frac{1}{2},-\frac{1}{16}\right) \otimes V_{\alpha, \beta}^{\prime}$ we can and only can get the highest weight modules with highest weight $\left(\frac{1}{2},-\frac{1}{2}\right),\left(\frac{1}{2}, 0\right)$ and $\left(\frac{1}{2},-\frac{1}{16}\right)$.

It is well known that in the physics literature that the conformal field theory associated to $V(1 / 2,0)$ has three simple modules $V(1 / 2,0)$, $V(1 / 2,-1 / 2)$ and $V(1 / 2,-1 / 16)$ and the fusion rules among these modules are given by

$$
\begin{aligned}
& V(1 / 2,-1 / 2) \times V(1 / 2,-1 / 2)=V(-1 / 2,0), \\
& V(1 / 2,-1 / 2) \times V(1 / 2,-1 / 16)=V(1 / 2,-1 / 16), \\
& V(1 / 2,-1 / 16) \times V(1 / 2,-1 / 16)=V(1 / 2,-1 / 2)+V(1 / 2,0),
\end{aligned}
$$

and $V(1 / 2,0)$ is the identity in the fusion rule relations. (See [DMZ]). From (III, IV, V) of Example 12, there seems to be some mysterious relations among these three modules.

Example 14. For any coprime $p, q \in\{2,3,4, \ldots\}$, set

$$
c_{p, q}=1-6 \frac{(p-q)^{2}}{p q} .
$$

From Theorem A in $\mathrm{A}$ we know that the maximal submodule $J(c, 0)$ of the Verma module $M(c, 0)$ is generated by $d_{-1} u$ if and only if $c \neq c_{p, q}$. 
In this example we assume that $c \neq c_{p, q}$. Then the irreducible module $V(c, 0)=M(c, 0) / J(c, 0)$ has a basis

$$
d_{-l}^{n_{l}} \cdots d_{-3}^{n_{3}} d_{-2}^{n_{2}} u, \quad n_{2}, n_{3}, \ldots, n_{l} \in \mathbb{Z}_{+} .
$$

If $\beta-\alpha=k \in \mathbb{Z}$, by Theorem 1 we know that $W=V(c, 0) \otimes V_{\alpha, \beta}^{\prime}$ has a smallest simple submodule $W^{(k-1)}$ which is generated by $u \otimes v_{i}, i \geq k$ and $W / W^{(k-1)}$ is a highest weight module of highest weight $(c, \beta-1)$.

Claim 1. $W / W^{(k-1)}$ is isomorphic to the Verma module $M(c, \beta-1)$.

Proof. It is enough to show that $x\left(u \otimes t^{k-1}\right) \notin W^{(k-1)}$ for all nonzero homogeneous element $x \in U\left(\mathrm{Vir}_{-}\right)$. Let's prove this by contradiction. Suppose $x\left(u \otimes t^{k-1}\right) \in W^{(k-1)}$ for some $x \in U\left(\mathrm{Vir}_{-}\right)_{-m}$ where $m \in \mathbb{Z}_{+}$.

Since $d_{-1} u=0$ and $d_{-1}\left(u \otimes t^{k}\right)=0$, then

$$
W^{(k-1)}=\sum_{i, l_{2}, \ldots, l_{s} \in \mathbb{Z}_{+}} \mathbb{C} d_{-s}^{l_{s}} \cdots d_{-3}^{l_{3}} d_{-2}^{l_{2}}\left(u \otimes t^{k+i}\right) .
$$

Now $x\left(u \otimes t^{k-1}\right) \in W^{(k-1)}$ has the following form:

$$
x\left(u \otimes t^{k-1}\right)=\sum_{\left(l_{2}, \ldots, l_{s}\right) \in I} a_{l_{2}, \ldots, l_{s}} d_{-s}^{l_{s}} \cdots d_{-3}^{l_{3}} d_{-2}^{l_{2}}\left(u \otimes t^{k+\left(\sum_{i=2}^{s} i l_{i}\right)-m-1}\right)
$$

where $I \subseteq\left(\mathbb{Z}_{+}\right)^{s-1}$ is a finite subset. Notice that $\left(\sum_{i=2}^{s} i l_{i}\right)-m-1 \in \mathbb{Z}_{+}$ for all $\left(l_{2}, \ldots, l_{s}\right) \in I$, that is, $\sum_{i=2}^{s} i l_{i} \geq m+1$.

On the other hand, we have $x\left(u \otimes t^{k-1}\right) \in \bigoplus_{i=0}^{m} V(c, 0)_{-i} \otimes t^{k-m-1}$. Let $\left(l_{2}, \ldots, l_{s}\right)$ be the index in $I$ such that $\sum_{i=2}^{s} i l_{i}$ is maximal, then we have

$$
\left(d_{-s}^{l_{s}} \cdots d_{-3}^{l_{3}} d_{-2}^{l_{2}} u\right) \otimes t^{k-m-1} \in \bigoplus_{i=0}^{m} V(c, 0)_{-i} \otimes t^{k-m-1},
$$

i.e., $\sum_{i=2}^{s} i l_{i} \leq m$ which is a contradiction.

We like to conclude our paper by pointing out that we are not able to determine the conditions for the quotient module $W^{(n-1)} / W^{(n)}$ in Remark 9 to be simple or to be a Verma module when it is not trivial. Our examples show that some of them are simple while some others are Verma modules. 


\section{Acknowledgments}

The research was carried out during the visit of the first and second authors at Wilfrid Laurier University in 2012 and 2013. They gratefully acknowledge the support (a short term grant) and hospitality of Wilfrid Laurier University. The second author is partially supported by NSF of China (Grant 11101380) and CSC of China (Grant 2010841037); the last author is partially supported by NSF of China (Grant 11271109) and NSERC. The authors want to thank Prof. R. Lu for a lot of helpful discussions when they were preparing the paper.

\section{REFERENCES}

[A] A. Astashkevich, On the structure of Verma modules over Virasoro and NeveuSchwarz algebras, Comm. Math. Phys. 186(3) (1997), 531-562.

$[\mathrm{CM}]$ C. Conley and C. Martin, A family of irreducible representations of the Witt Lie algebra with infinite-dimensional weight spaces, Compositio Math. 128(2) (2001), 153-175.

[DMS] P. Di Francesco, P. Mathieu and D. Sénéchal, Conformal field theory, Springer-Verlag, New York, 1997.

[DMZ] C. Dong, G. Mason and Y. Zhu, Discrete series of the Virasoro algebra and the moonshine module, Algebraic groups and their generalizations: quantum and infinite-dimensional methods (University Park, PA, 1991), 295-316, Proc. Sympos. Pure Math., 56, Part 2, Amer. Math. Soc., Providence, RI, 1994.

$[\mathrm{FF}]$ B. Feigin and D. Fuchs, Representations of the Virasoro algebra, Representation of Lie groups and related topics, 465-554, Adv. Stud. Contemp. Math. 7, Gordon and Breach, New York, 1990.

[FJK] E. Felinska, Z. Jaskolski and M. Kosztolowicz, Whittaker pairs for the Virasoro algebra and the Gaiotto-Bonelli-Maruyoshi-Tanzini states, J. Math. Phys. 53(3) (2012), 033504, 16pp.

[FZ] I. Frenkel and Y. Zhu, Vertex operator algebras associated to representations of affine and Virasoro algebras, Duke Math. J. 66(1) (1992), 123-168.

[GO] P. Goddard and D. Olive, Kac-Moody and Virasoro algebras in relation to quantum physics, Intemat. J. Modern Phys. A 1(2) (1986), 303-414.

[GLZ] X. Guo, R. Lu and K. Zhao, Fraction modules of the Virasoro algebra, Preprint, 2010.

[IK] K. Iohara and Y. Koga, Representation theory of the Virasoro algebra, Springer Monographs in Mathematics. Springer-Verlag London, Ltd., London, 2011.

[K] V. Kac, Contravariant form for infinite-dimensional Lie algebras and superalgebras, Lect. Notes in Phys. 94 (1979), 441-445.

[KR] V. Kac and A. Raina, Bombay lectures on highest weight representations of infinite-dimensional Lie algebras, Advanced Series in Mathematical Physics, 2. World Scientific Publishing Co., Inc., Teaneck, NJ, 1987.

[LLZ] G. Liu, R. Lü and K. Zhao, A class of simple weight Virasoro modules, Preprint, arXiv:1211.0998.

[LGZ] R. Lü, X. Guo and K. Zhao, Irreducible modules over the Virasoro algebra, Doc. Math. 16 (2011), 709-721.

[LZ] R. Lü and K. Zhao, Irreducible Virasoro modules from irreducible Weyl modules, Preprint, arXiv:1209.3746.

[M] O. Mathieu, Classification of Harish-Chandra modules over the Virasoro Lie algebra, Invent. Math. 107(2) (1992), 225-234. 
[MW] V. Mazorchuk and E. Wiesner, Simple Virasoro modules induced from codimension one subalgebras of the positive part, arXiv:1209.1691, to appear in Proc. AMS.

[MZ1] V. Mazorchuk and K. Zhao, Classification of simple weight Virasoro modules with a finite-dimensional weight space, J. Algebra 307(1) (2007), 209-214.

[MZ2] V. Mazorchuk and K. Zhao, Simple Virasoro modules which are locally finite over a positive part, Preprint, arXiv:1205.5937.

[MoP] R. V. Moody and A. Pianzola, Lie algebras with triangular decompositions, Canad. Math. Soc., Ser. Mono. Adv. Texts, A Wiley-Interscience Publication, John Wiley \& Sons Inc., New York, 1995.

[OW] M. Ondrus and E. Wiesner, Whittaker modules for the Virasoro algebra, J. Algebra Appl. 8(3) (2009), 363-377.

[Y] S. Yanagida, Whittaker vectors of the Virasoro algebra in terms of Jack symmetric polynomial, J. Algebra 333(1) (2011), 273-294.

[Z] H. Zhang, A class of representations over the Virasoro algebra, J. Algebra 190(1) (1997), 1-10.

[Zk] K. Zhao, Representations of the Virasoro algebra I, J. Algebra, 176(3)(1995), 882-907.

H.C.: School of Mathematical Sciences, University of Science and Technology of China, Hefei 230026, Anhui, P. R. China.

e-mail: hjchenmath@gmail.com.

X.G.: Department of Mathematics, Zhengzhou University, Zhengzhou 450001, Henan, P. R. China. e-mail: guoxq@zzu.edu.cn.

K.Z.: Department of Mathematics, Wilfrid Laurier University, Waterloo, ON, Canada N2L 3C5; and College of Mathematics and Information Science, Hebei Normal (Teachers) University, Shijiazhuang 050016, Hebei, P. R. China. e-mail: kzhao@wlu.ca. 